This item was submitted to Loughborough's Research Repository by the author.

Items in Figshare are protected by copyright, with all rights reserved, unless otherwise indicated.

\title{
Thickness-dependent surface energy and formation of epitaxial quantum dots
}

PLEASE CITE THE PUBLISHED VERSION

https://doi.org/10.1016/j.tsf.2020.138363

PUBLISHER

Elsevier BV

VERSION

AM (Accepted Manuscript)

\section{PUBLISHER STATEMENT}

This paper was accepted for publication in the journal Thin Solid Films and the definitive published version is available at https://doi.org/10.1016/j.tsf.2020.138363

\section{LICENCE}

CC BY-NC-ND 4.0

\section{REPOSITORY RECORD}

Lozovoy, Kirill A, Ying Zhou, Roger Smith, Adam Lloyd, Andrey P Kokhanenko, Vladimir V Dirko, Nataliya Yu Akimenko, Denis V Grigoryev, and Alexander V Voitsekhovskii. 2020. "Thickness-dependent Surface Energy and Formation of Epitaxial Quantum Dots”. Loughborough University. https://hdl.handle.net/2134/13222025.v1. 


\title{
Thickness-dependent surface energy and formation of epitaxial quantum dots
}

\author{
Kirill A. Lozovoy ${ }^{\mathrm{a}, *}$, Ying Zhou ${ }^{\mathrm{b}}$, Roger Smitha,b,**, Adam Lloyd ${ }^{\mathrm{b}}$, Andrey P. Kokhanenko ${ }^{\mathrm{a}}$, \\ Vladimir V. Dirko ${ }^{a}$, Nataliya Yu. Akimenko ${ }^{c}$, Denis V. Grigoryev ${ }^{\mathrm{a}}$, Alexander V. Voitsekhovskii ${ }^{\mathrm{a}}$ \\ ${ }^{a}$ Laboratory of Nanoelectronics and Nanophotonics, Tomsk State University, Lenin Ave, 36, Tomsk, 634050, Russia \\ ${ }^{b}$ School of Science, Loughborough University, LE11 3TU, UK \\ ${ }^{c}$ Pacific National University, Tihookeanskaya St, 136, Khabarovsk, 680035, Russia
}

\begin{abstract}
Numerous theoretical and experimental studies show that during epitaxial growth according to the Stranski-Krastanow mechanism in systems mismatched by the lattice constant, the change in the surface energy of the system during nucleation and further growth of quantum dots, plays the most important role. In particular, this factor determines the equilibrium and critical thicknesses of the transition from two-dimensional to three-dimensional growth, and also affects other kinetic characteristics of the ensemble of nanoclusters, including the nucleation rate, surface density, and average size of the islands. Recent theoretical studies have made it possible to determine that the surface energy in this process depends on the thickness of the material deposited on the substrate. In this paper, we construct a kinetic model of the formation and coherent growth of two-dimensional layers and quantum dots in mismatched epitaxial systems, taking into account the dependence of the specific surface energies on the thickness of the deposited material. In this approximation, we calculate the basic parameters of the formed array of nanoislands. Experimental studies were also carried out on the growth of two-dimensional layers and quantum dots of germanium on the silicon (100) surface. The results of experimental investigations confirm the proposed theoretical model.
\end{abstract}

Keywords: Two-dimensional materials, Quantum dots, Molecular beam epitaxy, Stranski-Krastanow growth mechanism

\section{Introduction}

Heterostructures with two-dimensional layers and quantum dots have taken their place as one of the main materials for creating nanoelectronics and photonic devices [1], for example, solar cells, visible and infrared photodetectors $[2,3,4]$, light-emitting devices $[5,6]$ and high-speed transistors $[7,8]$. In addition, these structures can be applied for modification of surfaces and the formation of coatings [9, 10]. They can be used for creation of textured surfaces, anti-reflection coatings [11] and implementation of photonic crystals and microresonators [12], as well as in quantum information technologies [13].

A most promising method for creating nanostructures with two-dimensional and zero-dimensional electron gas is molecular beam epitaxy. In this technology, for systems of two materials with a lattice mismatch, islands are formed on the surface of a two-dimensional wetting layer of growing material (Stranski-Krastanow mechanism) $[14,15]$.

Along with the energetically favourable process of relaxation of elastic stresses in nanoislands, the change in the surface energy of the system plays an important role in the transition from two-dimensional to

\footnotetext{
*Corresponding author: lozovoymailbox@gmail.com

** Corresponding author: r.smith@lboro.ac.uk
} 
three-dimensional growth [16]. The combination of these two factors determines such important quantities as the equilibrium and critical thicknesses of the two-dimensional wetting layer characterising the moment of transition from $2 \mathrm{D}$ to $3 \mathrm{D}$ growth $[17,18,19]$. In a theoretical analysis of the processes of nanostructure formation by the Stranski-Krastanow mechanism, it was usually assumed that the specific surface energies of the wetting layer and island faces are constant during growth [14, 16, 17, 18, $19,20,21]$. However, a number of theoretical studies show that these values depend on the thickness of the two-dimensional layer $[22,23,24,25,26,27]$. Namely, with an increase in the thickness of the 2D layer, the specific surface energies tend to their equilibrium value, which is characteristic of an unstrained material.

For instance, the results of energy calculations for the germanium/silicon (100) system show clearly that the surface energies of the $\mathrm{Ge}(100)$ and $\mathrm{Ge}(105)$ facets decrease with the the number of the deposited Ge layers. This effect is often considered as the reason of Ge pyramids formation on Si substrate [26].

Until now, the attempt to take into account the change in surface energies when modelling growth processes for the Stranski-Krastanow mechanism were made only within the framework of simpler thermodynamic models such as [28] and a generalisation [29] of the Muller-Kern theory [30, 31, 32]. Nevertheless, it allows one to determine only purely thermodynamic parameters of the system, such as, for example, the change in free energy, the critical size of an island, the nucleation barrier, and the equilibrium thickness. However, the most interesting properties are not thermodynamic but kinetic, which also depend on the dynamics of the deposition processes, in particular, on the growth rate. These include the surface density and the size distribution function of islands, as well as the critical thickness of the transition from two-dimensional to three-dimensional growth, which corresponds to the moment actually observed in the experiment when islands began to form.

In this work, we propose a kinetic model that takes into account the dependence of surface energies on the thickness, and refines the model previously proposed by Dubrovskii [33] and developed in our further works $[34,35]$. As a model system for calculations, we will use the classical system of germanium quantum dots on the silicon surface (100), in which, however, new unexpected phenomena continue to be discovered $[36,37,38,39,40]$.

\section{Theoretical model}

The change in the free energy of the substrate/growing material system during the transition of $i$ atoms from the wetting layer to an island without taking into account the dependence of surface energies on the amount of deposited material is written in the following form [14]:

$$
\Delta F(i)=\Delta F_{\text {surf }}(i)+\Delta F_{\text {elas }}(i)+\Delta F_{\text {attr }}(i),
$$

where $\Delta F_{\text {surf }}$ is the change in surface energy, $\Delta F_{\text {elas }}$ is the relaxation of elastic strains, and $\Delta F_{\text {attr }}$ is the decrease in the attraction of atoms to the substrate.

The dependencies of the specific surface energy of the wetting layer and the side facets of the pyramid on the thickness $h$ of the material deposited on the surface can be written in the general form as follows [29]:

$$
\begin{gathered}
\gamma(0, h)=\left[\gamma_{0}(0)-\gamma_{\infty}(0)\right] e^{-B_{0} \frac{h}{d_{0}}}+\gamma_{\infty}(0), \\
\gamma(\phi, h)=\left[\gamma_{0}(\phi)-\gamma_{\infty}(\phi)\right] e^{-B_{\phi} \frac{h}{d_{0}}}+\gamma_{\infty}(\phi),
\end{gathered}
$$

where $\gamma(0, h)$ and $\gamma(\phi, h)$ are specific surface energies of the base and side facets of the pyramid; $\phi$ is the angle between the side facet of an island and its base; $\gamma_{0}$ and $\gamma_{\infty}$ are the specific surface energies of the facet on the surface of the substrate (in the absence of the wetting layer) and on the surface of the strained two-dimensional layer of the deposited material (infinitely large thickness of the wetting layer), respectively; $B_{0}$ and $B_{\phi}$ are dimensionless parameters characterising the rate of change of specific surface energies with the thickness of the deposited material; $d_{0}$ is the thickness of one monolayer (ML) of the growing material.

The density of the wetting energy $\Psi_{0}$ will also depend on the thickness of the wetting layer: 


$$
\Psi_{0}(h)=\gamma_{s}-\left[\gamma_{0}(0)-\gamma_{\infty}(0)\right] e^{-B_{0} \frac{h}{d_{0}}}-\gamma_{\infty}(0),
$$

where $\gamma_{s}$ is the specific surface energy of the substrate material [41].

Then substituting explicit expressions for all three energy contributions into Eq. (1), taking into account the dependence of the specific surface energy on the thickness of the wetting layer $h$, we obtain the following final expression:

$$
\Delta F(i, h)=\frac{(\gamma(\phi, h) / \cos \phi-\gamma(0, h))}{k_{B} T} \alpha^{2} l_{0}^{2} i^{2 / 3}-\left\{[1-Z(\phi)] \lambda \epsilon_{0}^{2}-\frac{\Psi_{0}(h)}{d_{0}} \exp \left(-\frac{h}{k_{0} d_{0}}\right)\right\} \frac{l_{0}^{2} d_{0}}{k_{B} T} i,
$$

where $l_{0}$ is the average distance between atoms on the surface; $k_{B}$ is Boltzmann's constant; $T$ is the substrate temperature; $\alpha$ is a geometric factor depending on the shape of an island; $k_{0}$ is the wetting energy relaxation coefficient $[41] ; Z(\phi)$ is the elastic energy relaxation coefficient $[42,43,44] ; \lambda$ is the elastic modulus of the material; $\epsilon_{0}$ is the lattice mismatch.

The function (5) allows one to determine all the necessary thermodynamic parameters of the system. First, the equilibrium thickness of the wetting layer $h_{e q}$, at which the transition to the formation of threedimensional islands becomes possible, is found from the solution of the following equation:

$$
[1-Z(\phi)] \lambda \epsilon_{0}^{2}-\frac{1}{d_{0}}\left\{\gamma_{s}-\left[\gamma_{0}(0)-\gamma_{\infty}(0)\right] e^{-B_{0} \frac{h_{e q}}{d_{0}}}-\gamma_{\infty}(0)\right\} \exp \left(-\frac{h_{e q}}{k_{0} d_{0}}\right)=0 .
$$

Then, to find the critical number of atoms in an island $i_{c}$, at which the function (5) has a maximum, it is necessary to use the following expression:

$$
i_{c}(h)=\left(\frac{2(\gamma(\phi, h) / \cos \phi-\gamma(0, h)) \alpha^{2}}{3\left\{[1-Z(\phi)] \lambda \epsilon_{0}^{2}-\frac{\Psi_{0}(h)}{d_{0}} \exp \left(-\frac{h}{k_{0} d_{0}}\right)\right\} d_{0}}\right)^{3} .
$$

Finally, the nucleation activation barrier $\Delta F\left(i_{c}, h\right)$ is obtained by substituting the critical number of atoms $i_{c}$ calculated from Eq. (7) into Eq. (5) for change in the free energy of the system during the transition of atoms from the wetting layer to an island for a specific thickness of the deposited material $h$.

We now turn to the kinetic parameters characterising the time development of the growth process according to Stranski-Krastanow. The critical thickness of the transition from two-dimensional to threedimensional growth $h_{c}$ is determined by the material balance between the number of atoms entering the substrate/growing layer system from the flux and atoms passing from the wetting layer to the islands. This corresponds to the maximum thickness of the wetting layer $[14,45]$. This thickness may be evaluated by solving the following equation:

$$
\frac{4}{3 \sqrt{\pi}} \frac{h_{e q}^{2}}{d_{0}} \frac{\left(\frac{h_{c}}{h_{e q}}-1\right)}{2 a h_{c} \Delta F\left(i_{c}, h_{c}\right)}\left[\frac{2 \Delta F\left(i_{c}, h_{c}\right)}{\left(\frac{h_{c}}{h_{e q}}-1\right)^{2}} \frac{\tau}{t_{e q}}\right]^{\frac{5}{2}} \exp \left[\Delta F\left(i_{c}, h_{c}\right)\right]=1,
$$

where $\tau$ is the characteristic time of incorporating of adatom into a growing island $[14,35] ; t_{e q}=\frac{h_{e q}}{V}$ is the time of growth of 2D layer with the equilibrium thickness $h_{e q} ; V$ is the deposition rate.

The dependence of the nucleation rate of islands $I$ on the thickness of the wetting layer will be determined by the following formula:

$$
I(h)=\frac{a}{\tau l_{0}^{2}} \frac{h}{h_{e q}}\left(\frac{h}{h_{e q}}-1\right) e^{-\Delta F\left(i_{c}, h\right)} .
$$

The surface density of the islands at the end of the nucleation stage $N$, taking into account the dependence of surface energies on the amount of deposited material, will take the following form: 


$$
N(h)=\frac{a}{l_{0}^{2}} \frac{h_{c}}{h_{e q}} e^{-\Delta F\left(i_{c}, h_{c}\right)} \frac{\left(\frac{h_{c}}{h_{e q}}-1\right)^{2}}{2 \Delta F\left(i_{c}, h_{c}\right)} \frac{t_{e q}}{\tau}\left(1-e^{-e^{2 \Delta F\left(i_{c}, h_{c}\right) \frac{h-h_{c}}{h_{c}}}}\right) .
$$

Finally, the dynamics of changes in the size distribution function of islands with the thickness of twodimensional layer will be determined by the following expression:

$$
f(L, h)=\frac{2 L}{\left(\alpha l_{0}\right)^{2}} \frac{2 \Delta F\left(i_{c}, h_{c}\right) \tau}{\left(\frac{h_{c}}{h_{e q}}-1\right)^{2} t_{e q}} N \exp \left[\frac{2 \Delta F\left(i_{c}, h_{c}\right) \tau}{\left(\frac{h_{c}}{h_{e q}}-1\right)^{2} t_{e q}} \frac{L_{a v}^{2}(h)-L^{2}}{\left(\alpha l_{0}\right)^{2}}-e^{\frac{2 \Delta F\left(i_{c}, h_{c}\right) \tau}{\left(h_{e q}-1\right)^{2} t_{e q}} \frac{L_{a v}^{2}(h)-L^{2}}{\left(\alpha l_{0}\right)^{2}}}\right] .
$$

In addition, the calculations of surface energies for the case of growth of germanium on silicon (100) surface were performed via the Large-scale Atomic/Molecular Massively Parallel Simulator (LAMMPS) package [46] using the Tersoff potential [47]. Thickness-dependent surface energies $\gamma$ were calculated using the following expression $[26]$ :

$$
\gamma=\frac{E_{t o t}-N_{\mathrm{Si}} E_{\mathrm{Si}}-N_{\mathrm{Ge}} E_{\mathrm{Ge}}}{2 A},
$$

where $E_{\text {tot }}$ is the total energy of the system; $A$ is the surface area; $N_{\mathrm{Si}}$ and $N_{\mathrm{Ge}}$ are the number of $\mathrm{Si}$ and Ge atoms in the system and $E_{\mathrm{Si}}$ and $E_{\mathrm{Ge}}$ are cohesive energies of $\mathrm{Si}$ and Ge respectively. See our recent work [26] for details.

\section{Experimental details}

To verify the results of theoretical calculations, heterostructures with two-dimensional layers and quantum dots of germanium were grown on a silicon (100) surface.

The synthesis was carried out in a Katun-100 ultrahigh-vacuum molecular beam epitaxy unit equipped with two electron beam evaporators for silicon and germanium. The analytical part of the chamber consists of a mass spectrometer, a quartz thickness meter, an infrared pyrometer and a high energy electron diffractometer. During growth, diffraction patterns were recorded with a Full-HD camera. The growth rate of Ge was $0.03 \mathrm{ML} / \mathrm{s}(1 \mathrm{ML}=0.145 \mathrm{~nm})$. The temperature of the substrate was $500^{\circ} \mathrm{C}$. Silicon wafers with (100) crystallographic orientation and misorientation of less than $0.5^{\circ}$ were used as substrates. Before placing the wafers in the chamber, they were subjected to chemical treatment in order to create a thin continuous layer of silicon dioxide on the surface of the silicon wafer. After the plates were placed in a vacuum chamber, the substrate was annealed in a growth chamber at the temperature of $1000^{\circ} \mathrm{C}$ and silicon buffer layer was applied.

\section{Results and discussion}

The calculations were performed for the case of the growth of two-dimensional layers and quantum dots of germanium on the silicon surface (100). For this material system, the thermodynamic parameters used in the model have the following values $[26,33,41]: l_{0}=0.395 \mathrm{~nm}, d_{0}=0.145 \mathrm{~nm}, \lambda=1.27 \times 10^{11} \mathrm{~Pa}, k_{0}=0.8$, $\epsilon_{0}=0.042, \phi=11^{\circ}, Z(\phi)=0.7$.

Surface energies for facets of Ge pyramids formed on $\mathrm{Si}(100)$ with various contact angles were calculated. The contact angle of $11^{\circ}$ (i.e. (105) facet) proved to be the optimum orientation, that is confirmed by numerous experimental results $[10,14,27,48]$. Surface energies of (105) and (100) facets for various numbers of added Ge layers were determined. The surface energy of Ge(105) facet on the $\mathrm{Si}(100)$ surface was calculated to be $1.90,1.85$, and $1.80 \mathrm{~J} / \mathrm{m}^{2}$ after the addition of 1,2 and 3 monolayers. For 1 and 2 monolayers of germanium on silicon the value of Ge(100) surface energy is 1.97 and $1.93 \mathrm{~J} / \mathrm{m}^{2}$. The surface energies for clean $\mathrm{Si}(100)$ and $\mathrm{Ge}(100)$ surfaces are 2.27 and $1.87 \mathrm{~J} / \mathrm{m}^{2}$ respectively [26]. 
From the obtained values for specific surface energies, it can be seen that that this is energetically more favorable to form the (105) facets after the deposition of some germanium monolayers on the silicon surface $[26,48]$. Based on these calculated values, the dependences of surface energies on the thickness in the form (2) and (3) were constructed with the following parameters: $\gamma_{0}(0)=2.05 \mathrm{~J} / \mathrm{m}^{2}, \gamma_{\infty}(0)=1.87 \mathrm{~J} / \mathrm{m}^{2}$, $B_{0}=0.7, \gamma_{0}(\phi)=2.00 \mathrm{~J} / \mathrm{m}^{2}, \gamma_{\infty}(\phi)=1.80 \mathrm{~J} / \mathrm{m}^{2}, B_{\phi}=0.7$.

For the growth temperature $T=500^{\circ} \mathrm{C}$ and deposition rate $V=0.03 \mathrm{ML} / \mathrm{s}$ the solution of equations (6) and (8) using (2) and (3) gives for the equilibrium thickness of the wetting layer in the Ge/Si (100) system $h_{e q}=3.0 \mathrm{ML}$, and for the critical thickness of the transition from two-dimensional to three-dimensional growth $h_{c}=4.0 \mathrm{ML}$.

Fig. 1 presents graphs of the dependence of the change in free energy during the transition of atoms from the wetting layer to an island (5) on the size of the nucleus for the growth temperature $T=500^{\circ} \mathrm{C}$ and various thicknesses of deposited germanium. In addition, Fig. 1 shows the dependence of the critical nucleus size $i_{c}$ on the thickness of the germanium wetting layer (7).

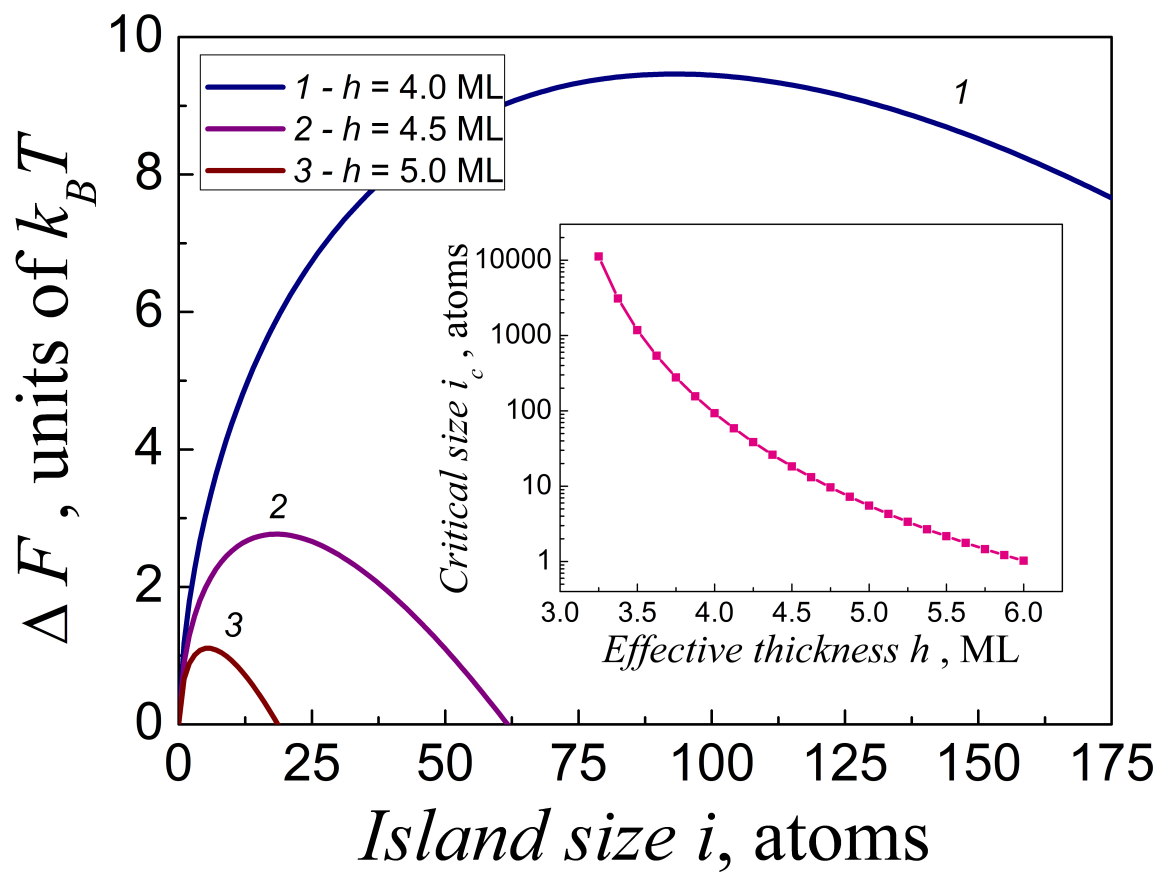

Figure 1: The free energy function $\Delta F(i, h)$ for the growth temperature $T=500^{\circ} \mathrm{C}$ and various thicknesses of Ge wetting layer $h$. Inset: The critical number of atoms in an island $i_{c}$ as a function of the Ge wetting layer thickness.

Fig. 1 shows that when the wetting layer reaches the critical thickness, the active formation of quantum dot nuclei begins, since the critical number of atoms and the energy barrier of nucleation rapidly decrease with an increase in the thickness of the two-dimensional layer from 4 to $5 \mathrm{ML}$. With the wetting layer thickness $h=4.0 \mathrm{ML}$, the maximum of the function defined in (5) is reached at the number of atoms in the nucleus $i_{c} \approx 100$ and its value is $\Delta F \approx 9 k_{B} T$. Already at the thickness $h=4.5 \mathrm{ML}$, the critical number of atoms is $i_{c} \approx 20$, and the activation barrier of nucleation is $\Delta F \approx 3 k_{B} T$ (Fig. 1 ). As the thickness of the wetting layer increases to $6 \mathrm{ML}$, the critical size of the nucleus tends to unity, i.e., each precipitated adatom forms an island. From a thermodynamic point of view the reason of the formation of 3D islands is that higher islands have a lower specific surface energy than the (100) surface.

Fig. 2 shows the effective amount of the deposited material without taking into account the nucleation 
of islands $h(1)$ and the thickness of the germanium wetting layer of germanium, taking into account the consumption of adatoms by growing islands (2). From Fig. 2 it is obvious that when the wetting layer reaches the critical thickness $h_{c}=4.0 \mathrm{ML}$, the active formation of islands begins due to the consumption of atoms of the wetting layer, which leads to a decrease in its actual thickness. Such a behaviour of the thickness of the wetting layer is also observed experimentally [14, 28, 41, 49].

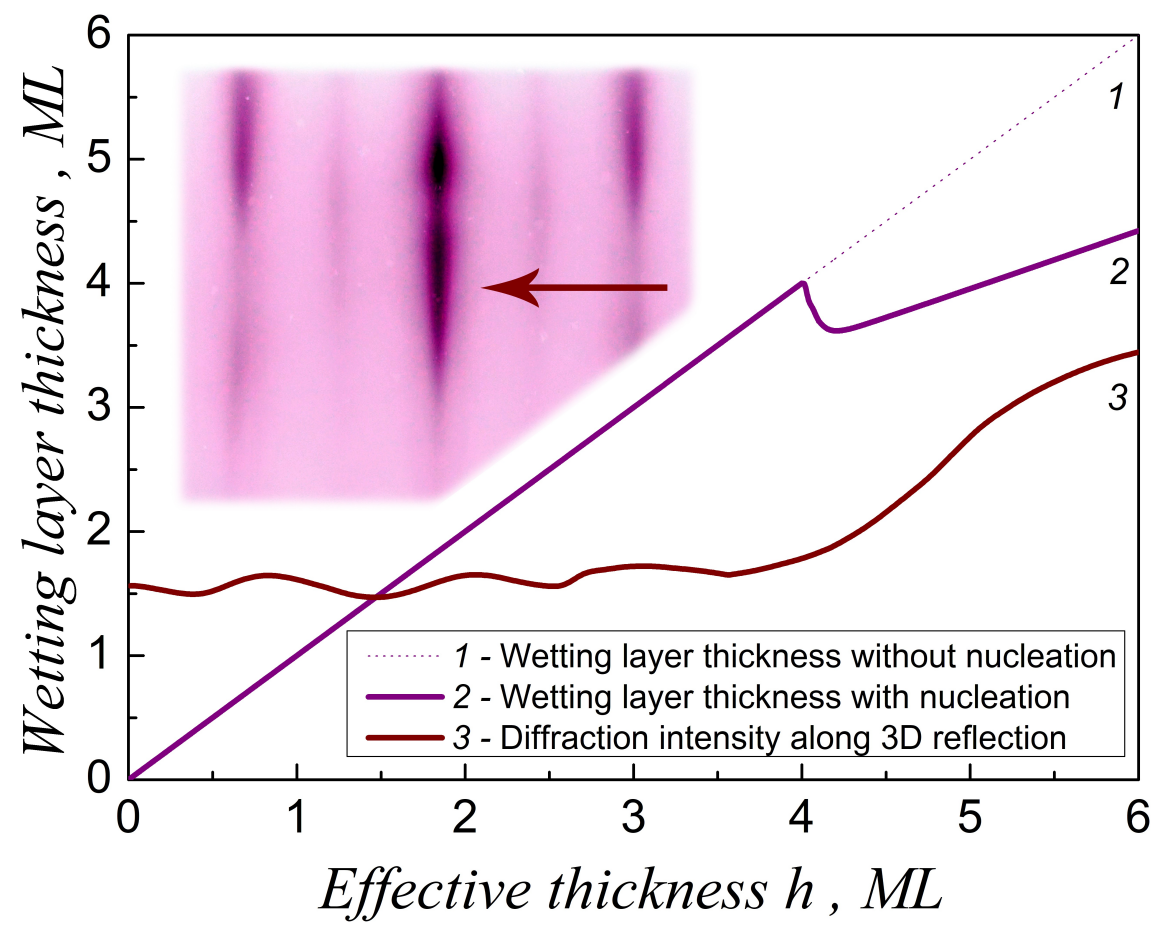

Figure 2: The effective amount of the deposited material without taking into account the nucleation of islands $h$ (1), the thickness of the germanium wetting layer, taking into account the consumption of adatoms by growing islands (2), and intensity profile (arbitrary units) of the diffraction pattern in [110] azimuth at the point of occurrence of the 3D reflection (3) for the growth temperature $T=500^{\circ} \mathrm{C}$ and deposition rate $V=0.03 \mathrm{ML} / \mathrm{s}$. Inset: diffraction pattern in [110] azimuth for the effective thickness $h=1.0 \mathrm{ML}$ (arrow indicates the area of appearance of 3D reflex).

In addition, Fig. 2 shows the experimentally obtained intensity profile of the diffraction pattern in [110] azimuth at the point of occurrence of the $3 \mathrm{D}$ reflex (3) for the growth temperature $T=500^{\circ} \mathrm{C}$ and deposition rate $V=0.03 \mathrm{ML} / \mathrm{s}$. The presence of intensity oscillations at the initial stage implies the realisation of a twodimensional mechanism of growth at this stage $[50,51,52,53]$. When the two-dimensional layer reaches the thickness of 4 monolayers, formation of three-dimensional islands occurs, which is observed by a characteristic change in the diffraction pattern and the termination of intensity oscillations (Fig. 2), which confirms the calculated critical thickness $h_{c}=4.0 \mathrm{ML}$ obtained within the framework of the proposed model.

Fig. 3 shows the thickness dependences of normalised values of nucleation rate $I$, surface density $N$ and average size of quantum dots $L_{a v}$ for the growth temperature $T=500^{\circ} \mathrm{C}$ and deposition rate $V=0.03 \mathrm{ML} / \mathrm{s}$. The islands nucleation rate is normalised to its maximum value of $1.0 \times 10^{14} \mathrm{~m}^{-2} \mathrm{~s}^{-1}$. The surface density of nanoclusters is normalised to its stationary value at the end of the nucleation stage $3.5 \times 10^{14} \mathrm{~m}^{-2}$. The average size of quantum dots is normalised to its final value $28 \mathrm{~nm}$ at the end of the growth process.

As shown in Fig. 3, the fastest process is the nucleation of islands. The islands nucleate most intensively 


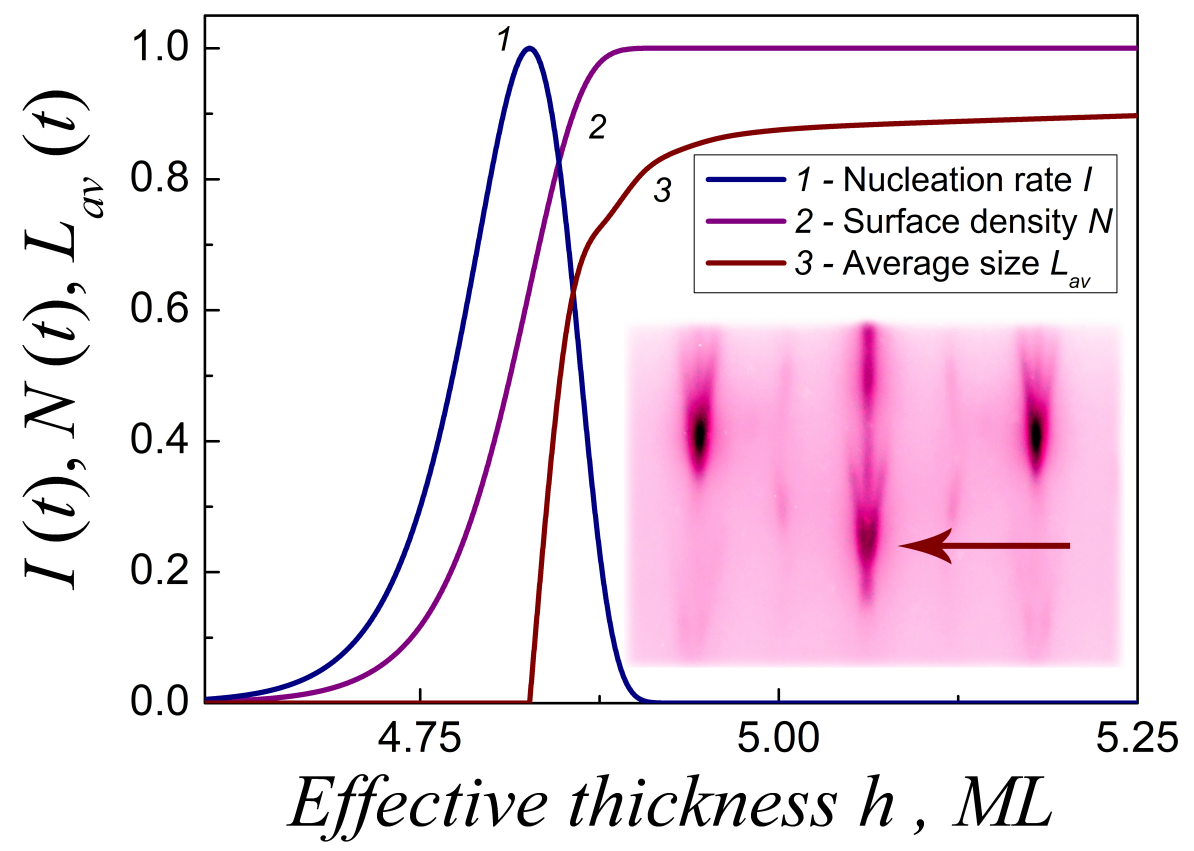

Figure 3: Thickness dependences of normalised values of nucleation rate $I$ (1), surface density $N$ (2) and average size of quantum dots $L_{a v}$ (3) for the growth temperature $T=500^{\circ} \mathrm{C}$ and deposition rate $V=0.03 \mathrm{ML} / \mathrm{s}$. Inset: The diffraction pattern along the [110] azimuth for the effective thickness $h=5.0 \mathrm{ML}$ (arrow indicates the area of 3D reflex).

in a very narrow range of effective thicknesses of the wetting layer from 4 to $5 \mathrm{ML}$. By the end of the nucleation stage, the surface density of the islands reaches its maximum stationary value. In the same interval of effective thickness the most pronounced increase of islands sizes takes places. At the same time the further growth of islands in size proceeds for significantly higher effective thicknesses, until the end of the deposition.

The insets to Figs. 2 and 3 show the experimentally obtained patterns of high energy electron diffraction for germanium synthesis on the silicon surface for the growth temperature $T=500^{\circ} \mathrm{C}$ and deposition rate $V=0.03 \mathrm{ML} / \mathrm{s}$ at two effective thicknesses of the deposited material: $h=1.0 \mathrm{ML}$ and $h=5.0 \mathrm{ML}$, respectively. The first picture corresponds to the processes of the formation of a two-dimensional wetting layer and has a traditional linear appearance (inset to Fig. 2). The second picture corresponds to the nucleation of pyramidal quantum dots with side facets (105) [54] and bright dot reflexes characterising the three-dimensional growth mode are clearly visible on it (inset to Fig. 3). Thus, the experimental studies on the synthesis of two-dimensional layers and quantum dots of germanium on a silicon surface (100) confirm the main conclusions of the developed model.

\section{Conclusions}

In this paper we generalise the kinetic model of the epitaxial growth by the Stranski-Krastanow mechanism taking into account the dependence of the specific surface energies of the facets of a three-dimensional island on the amount of deposited material. In this approximation, expressions are derived that allow one to determine the equilibrium and critical thicknesses of the wetting layer, nucleation rate, surface density and size distribution function of quantum dots at an arbitrary stage of growth. For the system of germanium 
quantum dots on the silicon (100) surface, the surface energies of the (100) and (105) facets forming the pyramidal nanocluster are calculated. The dependences of the main kinetic parameters of the system on the effective thickness of the deposited material are obtained. The calculated values are supported by the results of the experimental studies on the synthesis of two-dimensional layers and quantum dots of germanium on silicon.

\section{Declaration of Competing Interests}

The authors declare that they have no known competing financial interests or personal relationships that could have appeared to influence the work reported in this paper.

\section{Acknowledgements}

The reported study was funded by the Russian Science Foundation according to the research project No. 19-72-00019. Roger Smith would like to thank the Royal Society of London for an international exchange grant between Loughborough University and Tomsk State University.

\section{References}

[1] D. N. Basov, R. D. Averitt, D. Hsieh, Towards properties on demand in quantum materials, Nature Materials 16 (2017) $1077-1088$.

[2] I. I. Izhnin, O. I. Fitsych, A. V. Voitsekhovskii, A. P. Kokhanenko, K. A. Lozovoy, V. V. Dirko, Nanostructures with Ge-Si quantum dots for infrared photodetectors, Opto-Electronics Review 26 (2018) 195-200.

[3] S. Wirths, D. Buca, S. Mantl, Si-Ge-Sn alloys: from growth to applications, Prog. Cryst. Growth Charact. Mater. 62 (2016) $1-39$

[4] Y. Okada, N. J. Ekins-Daukes, T. Kita, R. Tamaki, M. Yoshida, a. Pusch, O. Hess, C. C. Phillips, D. J. Farrell, K. Yoshida, N. Ahsan, Y. Shoji, T. Sogabe, J.-F. Guillemoles, Intermediate band solar cells: Recent progress and future directions, Applied Physics Reviews 2 (2015) 021302.

[5] T. Davidson-Hall, H. Aziz, Perspective : Toward highly stable electroluminescent quantum dot light-emitting devices in the visible range, Applied Physics Letters 116 (2020) 010502.

[6] J. Wu, S. Chen, A. Seeds, H. Liu, Quantum dot optoelectronic devices: lasers, photodetectors and solar cells, Journal of Physics D 48 (2015) 363001.

[7] N. W. Hendrickx, D. P. Franke, A. Sammak, M. Kouwenhoven, D. Sabbagh, L. Yeoh, R. Li, M. L. V. Tagliaferri, M. Virgilio, G. Capellini, G. Scappucci, M. Veldhorst, Gate-controlled quamtum dots and superconductivity in planar germanium, Nature Communications 9 (2018) 2835.

[8] M. Schroter, T. Rosenbaum, P. Chevalier, B. Heinemann, S. P. Voinigescu, E. Preisler, J. Bock, A. Mukherjee, SiGe HBT technology: Future trends and TCAD-based roadmap, Proceedings of the IEEE 105 (2017) 1068-1086.

[9] I. I. Izhnin, K. R. Kurbanov, K. A. Lozovoy, A. P. Kokhanenko, V. V. Dirko, A. V. Voitsekhovskii, Epitaxial fabrication of 2D materials of group IV elements, Applied Nanoscience (2020). doi:10.1007/s13204-020-01372-4.

[10] J.-N. Aqua, I. Berbezier, L. Favre, T. Frisch, A. Ronda, Growth and self-organization of SiGe nanostructures, Physics Reports 522 (2013) 59-189.

[11] Y. Ota, D. Yurasov, A. Novikov, M. Shaleev, K. Gotoh, Y. Kurokawa, N. Usami, Impact of size distributions of Ge islands as etching masks for anisotropic etching on formation of anti-reflection structures, Japanese Journal of Applied Physics 58 (2019) 045505.

[12] V. Rutckaia, F. Heyroth, A. Novikov, M. Shaleev, M. Petrov, J. Schilling, Quantum dot emission driven by Mie resonances in silicon nanostructures, Nano Letters 17 (2017) 6886-6892.

[13] W. I. L. Lawrie, H. G. J. Eenink, N. W. Hendrickx, J. M. Boter, L. Petit, S. V. Amitonov, M. Lodari, B. P. Wuetz, C. Volk, S. Philips, G. Droulers, N. Kalhor, F. V. Riggelen, D. Brousse, A. Sammak, L. M. K. Vandersypen, G. Scappucci, M. Veldhorst, Quantum Dot Arrays in Silicon and Germanium, Applied Physics Letters 116 (2020) 080501.

14] V. G. Dubrovskii, Nucleation Theory and Growth of Nanostructures, Springer, Berlin, 2014.

[15] J. E. Prieto, I. Markov, Stranski-Krastanov mechanism of growth and the effect of misfit sign on quantum dots nucleation, Surface Science 664 (2017) 172-184.

[16] X. L. Li, C. X. Wang, G. W. Yang, Thermodynamic theory of growth of nanostructures, Progress in Materials Science 64 (2014) 121-199.

[17] A. I. Nikiforov, V. A. Timofeev, S. A. Teys, A. K. Gutakovsky, O. P. Pchelyakov, Initial stage growth of GexSi1-x layers and Ge quantum dot formation on GexSi1-x surface by MBE, Nanoscale research letters 7 (2012) 561. 
[18] K. A. Lozovoy, A. P. Kokhanenko, A. V. Voitsekhovskii, Critical thickness of transition from 2D to 3D growth and peculiarities of quantum dots formation in GexSi1-x/Sn/Si and Ge1-ySny/Si systems, Surface Science 669 (2018) 45-49.

[19] K. A. Lozovoy, A. G. Korotaev, A. P. Kokhanenko, V. Dirko, A. V. Voitsekhovskii, Kinetics of epitaxial formation of nanostructures by Frank-van der Merwe, Volmer-Weber and Stranski-Krastanow growth modes, Surface and Coatings Technology 384 (2020) 125289.

[20] K. Liu, I. Berbezier, T. David, L. Favre, A. Ronda, M. Abbarchi, P. W. Voorhees, J.-N. Aqua, Nucleation versus instability race in strained films, Physical Review Materials 1 (2017) 053402.

[21] F. Du, H. Huang, A generalized theory of thin film growth, Surface Science 669 (2018) 154-159.

[22] J. Tersoff, Stress-induced layer-by-layer growth of Ge on Si(100), Physical Review B 43 (1991) 9377-9380.

[23] L. G. Wang, P. Kratzer, M. Scheffler, N. Moll, Formation and stability of self-assembled coherent islands in highly mismatched heteroepitaxy, Physical Review Letters 82 (1999) 4042-4045.

[24] G.-H. Lu, M. Cuma, F. Liu, First-principles study of strain stabilization of Ge (105) facet on Si (001), Physical Review B $72(12)(2005) 125415$.

[25] D. Scopece, F. Montalenti, M. J. Beck, Stability of Ge on Si(1 1 10) surfaces and the role of dimer tilting, Physical Review B 85 (2012) 085312.

[26] Y. Zhou, A. Lloyd, R. Smith, K. A. Lozovoy, C. Scott, A. Voitsekhovskii, A. P. Kokhanenko, Molecular dynamics simulations of the growth of Ge on Si, Surface Science 696 (2020) 121594.

[27] K. Liu, I. Berbezier, L. Favre, A. Ronda, M. Abbarchi, P. Donnadieu, P. W. Voorhees, J.-N. Aqua, Capillary-driven elastic attraction between quantum dots, Nanoscale 11 (2019) 7798-7804.

[28] M. Brehm, F. Montalenti, M. Grydlik, G. Vastola, H. Lichtenberger, N. Hrauda, M. J. Beck, T. Fromherz, F. Schaffler, L. Miglio, G. Bauer, Key role of the wetting layer in revealing the hidden path of Ge/Si(001) Stranski-Krastanow growth onset, Physical Review B 80 (2009) 205321.

[29] K. A. Lozovoy, A. P. Kokhanenko, A. V. Voitsekhovskii, Generalized Muller-Kern formula for equilibrium thickness of a wetting layer with respect to the dependence of the surface energy of island facets on the thickness of the $2 \mathrm{D}$ layer, Physical Chemistry Chemical Physics 17 (2015) 30052.

[30] R. Kern, P. Muller, Three-dimensional towards two-dimensional coherent epitaxy initiated by surfactants, Journal of Crystal Growth 146 (1995) 193-197.

[31] P. Muller, R. Kern, The physical origin of the two-dimensional towards three-dimensional coherent epitaxial StranskiKrastanov transition, Applied Surface Science 102 (1996) 6-11.

[32] R. Kern, P. Muller, Elastic relaxation of coherent epitaxial deposits, Surface Science 392 (1997) $103-133$.

[33] V. G. Dubrovskii, G. E. Cirlin, V. M. Ustinov, Kinetics of the initial stage of coherent island formation in heteroepitaxial systems, Physical Review B 68 (2003) 075409.

[34] K. A. Lozovoy, A. P. Kokhanenko, A. V. Voitsekhovskii, Comparative analysis of germanium-silicon quantum dots formation on $\mathrm{Si}(100), \mathrm{Si}(111)$ and $\mathrm{Sn} / \mathrm{Si}(100)$ surfaces, Nanotechnology 29 (2018) 054002.

[35] K. A. Lozovoy, A. P. Kokhanenko, V. Dirko, N. Y. Akimenko, A. V. Voitsekhovskii, Evolution of epitaxial quantum dots formed by Volmer-Weber growth mechanism, Crystal Growth and Design 19 (2019) 7015-7021.

[36] A. A. Shklyaev, A. E. Budazhapova, Critical conditions for SiGe island formation during Ge deposition on Si(100) at high temperatures, Materials Science in Semiconductor Processing 57 (2017) 18-23.

[37] T. David, K. Aqua, J.-N. Liu, L. Favre, A. Ronda, M. Abbarchi, J.-B. Claude, I. Berbezier, New strategies for producing defect free sige strained nanolayers, Scientific Reports 8 (2018) 2891.

[38] S. Wang, N. Zhang, P. Chen, L. Wang, X. Yang, Z. Jiang, Z. Zhong, Toward precise site-controlling of self-assembled Ge quantum dots on Si microdisks, Nanotechnology 29 (2018) 345606.

[39] I. I. Izhnin, O. I. Fitsych, A. V. Voitsekhovskii, A. P. Kokhanenko, K. A. Lozovoy, V. V. Dirko, Interaction between islands in kinetic models of epitaxial growth of quantum dots, Applied Nanoscience (2019). doi:10.1007/s13204-019-00965-y.

[40] K. Liu, I. Berbezier, L. Favre, A. Ronda, T. David, M. Abbarchi, P. Gaillard, T. Frisch, B. Croset, J.-N. Aqua, Selforganization of sige planar nanowires via anisotropic elastic field, Physical Review Materials 3 (2019) 023403.

[41] A. V. Osipov, F. Schmitt, S. A. Kukushkin, P. Hess, Stress-driven nucleation of coherent islands: theory and experiment, Applied Surface Science 188 (2002) 156-162.

[42] C. Ratsch, A. Zangwill, Equilibrium theory of the Stranski-Krastanov epitaxial morphology, Surface Science 293 (1993) $123-131$.

[43] V. G. Dubrovskii, N. V. Sibirev, X. Zhang, R. A. Suris, Stress-driven nucleation of three-dimensional crystal islands: from quantum dots to nanoneedles, Crystal Growth and Design 10 (2010) 3949-3955.

[44] X. Zhang, V. G. Dubrovskii, N. V. Sibirev, X. Ren, Analytical study of elastic relaxation and plastic deformation in nanostructures on lattice mismatched substrates, Crystal Growth and Design 11 (2011) 5441-5448.

[45] A. I. Nikiforov, V. A. Cherepanov, O. P. Pchelyakov, A. V. Dvurechenskii, A. I. Yakimov, In situ RHEED control of self-organized Ge quantum dots, Thin Solid Films 380 (2000) 158-163.

[46] S. Plimpton, P. Crozier, A. Thompson, LAMMPS-large-scale atomic/molecular massively parallel simulator, Sandia National Laboratories 18 (2007) 43.

[47] J. Tersoff, Modeling solid-state chemistry: Interatomic potentials for multicomponent systems, Physical Review B 39 (8) (1989) 5566.

[48] S. A. Teys, Different growth mechanisms of Ge by Stranski-Krastanow on Si (111) and (001) surfaces: An STM study, Applied Surface Science 392 (2017) 1017-1025.

[49] R. Bergamaschini, M. Brehm, M. Grydlik, T. Fromherz, G. Bauer, F. Montalenti, Temperature-dependent evolution of the wetting layer thickness during Ge deposition on $\mathrm{Si}(001)$, Nanotechnology 22 (2011) 285704.

[50] A. I. Nikiforov, V. I. Mashanov, V. A. Timofeev, O. P. Pchelyakov, H.-H. Cheng, Refelection high energy electron diffraction 
studies on SixSnyGe1-x-y on $\mathrm{Si}(100)$ molecular beam epitaxial growth, Thin Solid Films 557 (2014) 188-191.

[51] A. Grimm, A. Fissel, E. Bugiel, T. F. Wietler, In situ observation of low temperature growth of Ge on $\operatorname{Si}\left(\begin{array}{lllllll}1 & 1\end{array}\right)$ by reflection high energy electron diffraction, Applied Surface Science 370 (2016) 40-48.

52] V. Timofeev, A. Nikiforov, A. Yakimov, V. Mashanov, I. Loshkarev, A. Bloshkin, V. Kirienko, V. Novikov, K. Kareva, Studying the morphology, structure and band diagram of thin GeSiSn films and their mid-infrared photoresponse, Semiconductor Science and Technology 34 (2019) 014001.

[53] V. S. Lysenko, S. V. Konadratenko, Y. N. Kozyrev, V. P. Kladko, O. Y. Gudymenko, V. V. Strelchuk, A. S. Nikolenko, O. S. Kondratenko, S. A. Iliash, G. S. Pekar, Morphology and optical properties of Ge nanocrystalline films grown by nonequilibrium epitaxy on $\mathrm{Si}(001)$ surface, Thin Solid Films 654 (2018) 54-60.

[54] A. A. Shklyaev, K. Romanyuk, S. Kosolobov, Surface morphology of Ge layers epitaxially grown on bare and oxidized $\mathrm{Si}(001)$ and $\mathrm{Si}(111)$ substrates, Surface Science 625 (2014) 50-56. 